\title{
tic\&société
}

Vol. $1, n^{\circ} 1 \mid 2007$

De TIS à tic\&société : dix ans après

\section{Retour sur une (d)ébauche : Une problématique communicationnelle du changement technique}

\section{Thierry Bardini}

\section{(2) OpenEdition \\ Journals}

Édition électronique

URL : http://journals.openedition.org/ticetsociete/245

DOI : $10.4000 /$ ticetsociete. 245

Éditeur

Association ARTIC

\section{Référence électronique}

Thierry Bardini, « Retour sur une (d)ébauche : Une problématique communicationnelle du changement technique », tic\&société [En ligne], Vol. 1, $n^{\circ} 1$ | 2007, mis en ligne le 15 février 2008, consulté le 14 novembre 2019. URL : http://journals.openedition.org/ticetsociete/245 ; DOI : 10.4000/ticetsociete. 245 


\section{Retour sur une (d)ébauche : Une problématique communicationnelle du changement technique}

Thierry BARDINI, Professeur agrégé

Département de communication

Université de Montréal

Adresse postale :

Département de Communication

Université de Montréal

C.P. 6128, Succursale Centre-ville

Montréal, QC

H3C $3 J 7$

Canada

Adresse de courriel :

thierry.bardini@umontreal.ca

Ingénieur agronome (ENSA Montpellier, 1986) et docteur en sociologie (Paris X, 1991), Thierry Bardini est professeur agrégé au Département de Communication à l'Université de Montréal. Ses intérêts de recherche couvrent l'ensemble de la cyberculture, depuis la création et l'usage des technologies de l'information et de la communication jusqu'à la biologie moléculaire. II a publié son premier livre, intitulé Bootstrapping: Douglas Englebart, Coevolution and the Genesis of Personal Computing, aux presses de I'Université Stanford en 2000. Son deuxième manuscrit, intitulé Junkware: The Disaffected Subject est actuellement sous évaluation aux éditions Semiotext(e), pour une parution en 2009 (http://www.junkware.net). 
Retour sur une (d)ébauche :

Une problématique communicationnelle du changement technique

\title{
Résumé
}

Je revisite ici mon papier paru en 1993 dans Technologie de l'Information et Société sous le titre "Diffusionnisme, constructivisme et modèle technique : ébauche d'une approche communicationnelle du changement technicosocial ». Je commence par envisager de nouveau les conditions de possibilité d'une synthèse de la théorie de la diffusion des innovations avec les théories (constructivistes) de la genèse des objets techniques, avant de revenir sur les critiques et apories propres à l'une de ces dernières, la théorie de l'acteur-réseau. Plus qu'à une résolution de ces problèmes, j'insiste au contraire sur quelques pistes problématiques, en espérant que certaines apories théories, plutôt qu'obstacles à, peuvent devenir moteur de, la réflexion.

Mots-clés : Changement technique, innovation, diffusion, théorie, médiation, acteur-réseau.

\begin{abstract}
I revisit here my paper published in 1993 in Technologies de l'Information et Société, and entitled "Diffusionism, constructivism and technological frame: A sketch of a communication approach of socio-technological change." I start with a new look at the conditions of possibility of a theoretical synthesis of the diffusion of innovations with the (constructivist) theories of the genesis of technological objects, before coming back to the aporia and critics of one of these theories, i.e. the actor-network theory (ANT). More than a solution to those problems, I insist here on some avenues for further thinking, hopeful that some theoretical dead-ends, rather than being only obstacles, can also be the engine of thought.
\end{abstract}

Keywords: technological change, innovation, diffusion, theory, mediation, actor-network. 
Thierry BARDINI

\section{Resumen}

Revisto aquí mi articulo publicado en 1993 por Technologies de l'Information et Société y titulado "Diffusionismo, constructivismo y modelo técnico: un ensayo de una visión communicational del cambio técnico". Empiezo con une nueva vista de las condiciones de posibilidad de una síntesis teorética de la teoría de la difusión de las innovaciones con las teorías (constuctivista) de la génesis de los objetos técnicos, antes de regresar a las aporías y criticas de una de ellas, o sea la teoría del actor-red. En lugar de tratar de resolver esos problemas, trato aquí de presentar nuevas avenidas de reflexión, esperando que las aporías teorética en lugar de obstáculos, se pueden cambiar en motores del pensamiento.

Palabras-clave: cambio técnico, innovación, difusión, teoría, mediación, actor-red. 
Retour sur une (d)ébauche :

Une problématique communicationnelle du changement technique Le philosophe «généreux » oublie à ses
dépens que d'un système, seules survivent les
vérités nuisibles.

- Emil Cioran, Syllogismes de l'amertume

Lorsque la nouvelle équipe de tic\&société, en la personne de Florence Millerand, m'a «passé commande » pour le présent papier, j'ai tout de suite ressenti un plaisir mêlé d'angoisse. Plaisir d'apprendre que mon article d'origine, intitulé « Diffusionnisme, constructivisme et modèle technique : Ébauche d'une approche communicationnelle du changement technico-social », pouvait être choisi dans le groupe des articles marquant la première vie de cette revue... mais angoisse aussi, d'avoir à revisiter une contribution datée, montrant certainement tous les signes de l'immaturité qui me caractérisait alors.

Ce papier a en effet marqué le début de ma carrière de professeur en communication, puisqu'il reprenait une présentation orale donnée lors de mon entretien d'embauche au département de communication de l'université de Montréal. Jeune sociologue, je venais de terminer mon stage post-doctoral avec Everett M. Rogers à l'Annenberg School for Communication de l'Université de Californie du Sud (USC). Je n'avais alors publié qu'un seul papier, qui démontrait plus de signes de ma formation antérieure en sociologie rurale qu'une quelconque compréhension de ce que la « communication » pouvait bien signifier. J'avais donc débuté mon sous-titre par le mot « ébauche » avec tout le respect possible, mais aussi en pleine conscience de mon incompétence en la matière.

Le jour de l'entretien d'embauche, j'avais commencé ma conférence sous les éclats de rire. Lapsus freudien s'il en était, mon premier acétate projetait une certaine confusion: un « $d$ » malin s'était glissé là où il n'aurait pas dû, transformant mon ébauche en quelque chose de beaucoup moins avouable. Depuis ce jour, j'ai souvent revu en moi et chez des étudiants en qui je croyais me reconnaître, ce qui mérite ce « $d$ », cette tentation d'aplanir les différences épistémologiques inconciliables, de fédérer par la pensée ce que la pratique rend pourtant difficilement envisageable avec le même équipement conceptuel. Bref, ce qui me pose encore problème lorsque je relis ce papier, presque quinze ans plus tard, c'est son manque de prise en compte d'un certain nombre 


\section{Thierry BARDINI}

d'apories constitutives de chacune des théories que j'essayai alors de réunir. Mais alors, plaiderais-je pour l'impossibilité d'une telle synthèse, et renierais cet article? Non, point trop n'en faut. Dans ce qui suit, je commencerai plutôt par expliciter les conditions de possibilité de la synthèse théorique originellement envisagée : à condition, d'abord, que les apories deviennent « moteur plutôt que frein ».

\section{Une synthèse éternellement à refaire}

La thèse principale de mon article de 1993 tiendrait finalement aujourd'hui dans cette proposition : les représentations des usagers potentiels ou actuels d'un changement technique participent à la production dudit changement. À l'aide d'un concept bifide de "représentation », signifiant à la fois le résultat d'opérations de délégation et le contenu cognitif qui les rendent possible, j'entendais réconcilier le modèle de la traduction et le modèle de la diffusion des innovations. Étant donné que ces deux modèles ne s'appliquent pas à expliciter la même phase du cycle de vie d'une innovation - conception pour le premier, adoption pour le second - je voyais une possibilité de synthèse justifiée par le recours à l'objet. En clair, ces deux modèles m'apparaissaient incomplets dans leur explicitation des phénomènes du changement technique. L'innovation, pensai-je alors, est une et indivisible, en expliciter la genèse ou la diffusion, ne rend compte que de la moitié de ses phénomènes. La présence de deux concepts transversaux communs à ces deux approches, le réseau et la médiation, me permettait d'espérer les réunir dans une approche synthétique des phénomènes du changement technique. Le fait que ces concepts référent « au domaine de la communication » me permettait de qualifier mon modèle de « communicationnel ». Sur la base de processus communicationnels à expliciter (i.e. de médiations), la conception anticipe l'adoption, et l'adoption récapitule la conception (comme on dit que l'ontogenèse récapitule la phylogenèse, ce qui est, par ailleurs, une erreur). Ces processus se cristallisent dans les représentations de l'usage et des usagers, et donnent ainsi un site stratégique où étudier de manière synthétique les phénomènes de changement technicosocial.

Dans mon esprit, cette proposition synthétique permettait de remédier aux principales carences de chacun des deux modèles considérés. II permettait d'une part de revenir sur la nature tautologique de la théorie de la diffusion des innovations, et, d'autre part, de faire sortir la théorie de l'acteur-réseau de l'impasse que constitue une sociologie de la controverse lorsqu'il s'agit d'expliciter des phénomènes, qui, justement, ne donnent pas (ou plus) matière à controverse. Un seul mouvement réunissait ainsi ces deux « remèdes », le refus 
Retour sur une (d)ébauche :

Une problématique communicationnelle du changement technique

du déterminisme, qu'il soit technique ou social—ce qui est devenu depuis lors une sorte de cliché, et ce qui ancrait définitivement mon texte dans l'air du temps... Oui, vous avez bien lu: ancré dans l'air ! Le refus du déterminisme technique mettait mon modèle à l'abri des pièges du biais pour l'innovation, et promettait de trouver une explication plus puissante que celles qui avaient recours à la notion de « propension pour l'innovation » [innovativeness] ou toute autre variable psychologisante du type «sensibilité à l'influence ». Le refus du déterminisme social, quant à lui, permettait d'envisager l'innovation au sein d'une histoire englobante, tant d'un point de vue social que technique.

Cette synthèse théorique passait cependant sous silence les incompatibilités mutuelles des deux théories, pour ne pas dire leur incommensurabilité. Et ce, non seulement au niveau de leur objet, c'est-à-dire de phases distinctes d'un même processus englobant (car cela militait plutôt pour la possibilité d'une synthèse), mais bien plutôt d'un point de vue épistémologique. Au sens de l'incommensurabilité paradigmatique envisagée par Thomas Kuhn dans La structure des révolutions scientifiques. Comment, en fait, imaginer concilier dans le même modèle un point de vue fonctionnaliste (pour ne pas dire positiviste) avec un point de vue constructiviste (pour ne pas dire relativiste) ? Comment sortir de l'impasse sans, en fin de compte, choisir son camp, et relire ou réinterpréter un modèle, contre son épistémologie, à l'aide de l'autre ? N'est-ce pas finalement ce que j'ai fait, en réinterprétant la théorie de la diffusion des innovations à l'aide de la théorie l'acteur réseau ? Beaucoup d'éléments dans mon texte le laissaient penser. La critique du diffusionnisme qui y était exposée recoupe celle qu'en faisaient les théoriciens de la traduction : réification de l'innovation, linéarité du modèle proposé, caractère a posteriori de la démarche, etc. On peinerait, à l'inverse, à y trouver une critique de la traduction depuis le point de vue de la théorie de la diffusion. Mais une question cruciale restait: d'un point de vue épistémologique, comment comprendre et décrire les réseaux de la diffusion à l'aide du concept d'acteur-réseau ? Et, de manière encore plus cruciale, comment traduire méthodologiquement cette convergence conceptuelle?

Autant d'apories, j'en ai peur, qui ne pouvaient mener, compte tenu de l'épistémologie constructiviste que j'épousai alors, qu'à un seul constat : cette synthèse, n'a finalement qu'un seul intérêt, celui de pouvoir problématiser la question du changement technico-social, sans avoir recours à une théorie toute faite, avant les faits. Suivre les acteurs, encore une idée dans l'air du temps ! 


\section{Thierry BARDINI}

Une conclusion logique aurait dû s'imposer: cette synthèse théorique n'a aucune valeur théorique, si l'on entend par là systématisme ou capacité à anticiper. Bref, l'accouplement de deux éléphants théoriques n'accouchait pratiquement que d'une souris méthodologique. Exercice de conceptualisation problématique, la synthèse théorique que j'amorçai n'aurait finalement d'intérêt que si elle était éternellement « remise sur le métier. »C'est justement ce à quoi je me suis ingénié par la suite, dans les quelques années qui ont suivi la publication de ce texte, finalement plus programmatique qu'autre chose.

\section{Des critiques de la traduction}

Alors même que je préparai mon texte pour TIS, de nombreuses voix commençaient à se faire entendre depuis l'intérieur de la forteresse paradigmatique constructiviste. La critique dont j'avais en quelque sorte fait l'économie dans ma tentative de synthèse me rattrapait donc. Je ne peux ici faire justice à ce foisonnement de la littérature qui m'a par la suite fortement inspiré, et je me bornerai donc à en brosser un portrait à grands traits, entre 1992 et 1996. Durant ces quelques années, plusieurs brèches théoriques et méthodologiques ont en effet été ouvertes dans ce qui paraissait auparavant être un édifice stable et cohérent.

La première de ces brèches a consisté en d'intenses discussions au sujet de la symétrie généralisée proposée par les théoriciens de l'acteur-réseau (Callon et Latour, en particulier) et rejetée par certains constructivistes anglais (et notamment Harry Collins). Callon et Latour proposaient d'étendre le principe de symétrie du programme fondateur, tel que proposé par David Bloor dès 1976. Ce principe s'exprime ainsi : "le même type d'explication devrait être utilisé pour tous les éléments qui composent un réseau hétérogène, que ces éléments soient des appareils, des forces naturelles ou des groupes sociaux" (Law, 1987, p.130). Le refus du "Grand Partage" (Latour, 1983) s'est traduit progressivement dans la pensée des membres de l'école de la traduction par l'introduction d'une nouvelle catégorie à l'analyse : l'actant. Pour sortir de l'impasse où nous a acculé la sociologie classique, disent en substance Latour et ses collègues, il faut reconnaître la capacité d'action à toutes les entités actives dans un narratif, qu'elles soient humaines ou non. D'autres s'y opposaient, parfois férocement. La publication, en 1992, de l'ouvrage collectif Science as Culture and as Practice, sous la direction d'Andrew Pickering, a permis à ces positions de s'exprimer, et aux principaux protagonistes de se fâcher à jamais. Pour ma part, j'ai vu dans cette controverse tout le caractère problématique de la position de l'acteur-réseau pour une tentative de concilier les réseaux (humains) de la diffusion des innovations avec les (acteur)réseaux constructivistes. 
Retour sur une (d)ébauche :

Une problématique communicationnelle du changement technique

La deuxième de ces brèches est apparue elle aussi dans les échanges musclés publiés dans l'ouvrage dirigé par Pickering. Collins et son collègue Yearley ne s'y étaient pas seulement pris à Callon et Latour, mais aussi à une autre «aile » du mouvement constructiviste représentée, entre autres, par Malcolm Ashemore et Steve Woolgar, et qualifiée de réflexiviste. Ceux-ci insistaient sur l'importance cruciale d'une réflexion sur la question baptisée par Ashmore d'argument du «tu quoque ». En son essence, cet argument retourne l'argument constructiviste : si vous dites que la science (ou la technique) est une construction sociale, en quoi votre propre description pourrait-elle alors échapper à la même caractérisation ? Apparaît alors le spectre de la régression infinie-du type construction sociale de la construction sociale de la science, ad infinitum. Les réponses proposées, après le morceau de bravoure représenté par la propre thèse d'Ashemore (1989), m'ont paru le plus souvent oiseuses... discussions à n'en plus finir sur les moyens à mettre en œuvre « pour que nos lecteurs ne nous croient pas trop », mises en œuvres de stratégies littéraires, etc. Quinze ans plus tard, mon impression n'a pas changé. Lors d'un séminaire à Bath, j'en étais même arrivé à proposer à Malcolm de collaborer avec des écrivains s'il voulait réellement faire progresser la question. Lorsque j'avais évoqué une solution analogue à Bruno Latour, deux ans plus tôt, mais en proposant cette fois de collaborer avec des scientifiques ou des ingénieurs, il m'avait répondu, très sérieusement, «vous n'y pensez pas, mon cher Bardini, ces gens sont nos drosophiles ! » Autant pour le relativisme réflexif...

La troisième de ces brèches a dévoilé un véritable angle mort de mon texte. Dès 1993, un texte fondamental de Madeleine Akrich avait montré les problèmes que l'usage posait aux analyses en termes d'acteur réseau. Depuis, de multiples contributions sont revenues sur cette question, et en particulier en ce qui concerne l'usage des médias (e.g. Chambat, 1994a et b ; Vedel, 1994). Certains, comme Florence Millerand (1999), ont proposé de compléter le tableau esquissé dans mon article en ajoutant le champ des théories de l'appropriation à celle de la construction et de la diffusion des innovations. J'ai pris bonne note de ces contributions qui restent néanmoins centrées sur des questions à la périphérie de mes intérêts de recherche. Je conçois cependant que cette critique soit fondamentale pour toute visée systématique sur l'innovation: conception et adoption n'ont finalement de sens que vis-à-vis d'usages effectifs. La linéarité de cette séquence est à la fois triviale et absolument problématique, et en particulier lorsqu'on s'intéresse aux phénomènes de re-invention (Rice et Rogers, 1980), d'usage de pointes (von 
Hippel, 1986), ou de substitution d'usage. Dans le cadre des études médiatiques à l'heure de la convergence tant annoncée, ces questions ne sauraient être sous-estimées.

La quatrième et dernière brèche que j'évoquerai ici est certainement celle qui m'a le plus occupé (et préoccupé). A peu près à la même époque, plusieurs voix se sont élevées pour dénoncer le «tournant sémiotique » pris par la théorie de l'acteur réseau (e.g. Lenoir, 1992). En recyclant de manière finalement assez superficielle le vocabulaire actanciel de Greimas, disaient ces critiques, l'acteur réseau réduit tout au texte, au vocabulaire du script. En faisant converger cette critique avec celle de la réflexivité, on concevra aisément que les «solutions proposées » n'ont souvent fait qu'empirer le mal, en donnant toute l'apparence d'arbitraire à la position constructiviste (cf infra, la crise Sokal). Comme le confessait Bruno Latour dès 1993, « l'absence d'une théorie de l'énonciation » minait les fondations mêmes de la théorisation de l'acteur-réseau. C'est ici aussi, pour ce que j'en comprends, que font sens les travaux de « l'École de Montréal » en communication organisationnelle, autour des recherches de mon cher collègue Jim Taylor, prolongées, entre autres, par celles de François Cooren et Daniel Robichaud (1996). Je ne suis cependant pas convaincu qu'ils aient pour l'instant résolu le problème qui se pose pour l'acteur-réseau, ou qu'ils ne puissent jamais le faire. II y a des apories qui résistent... Par un choix méthodologiquement judicieux mais théoriquement suicidaire, j'ai bien peur que les constructivistes adeptes du tournant sémiotique se soient placés dans l'incapacité d'envisager les rapports signe/monde qui déterminent en dernière instance la plausibilité de ces systèmes de signes que sont les « scripts » qu'ils produisent.

\section{Modes de présence et affordances}

J'ai cherché dans une autre direction pour ma part (mais pour le même résultat, j'en ai peur). Dans un texte datant de 1996 qui préfigure le présent article en une tentative de parfaire l'ébauche originelle de mon programme de recherche, j'ai proposé d'introduire les notions de virtualité et d'affordance (difficilement traduisible en français, même si certains ont proposé d'avoir recours au néologisme «promission »). L'introduction de cette dernière notion visait à réintroduire la matérialité dans l'analyse, en la distinguant de l'inscription: Alors que l'inscription suppose un "scribe" (en l'occurrence l'ingénieur, le designer, le technicien, bref, le concepteur), un auteur, la notion d'affordance traduit une possibilité pour. Un dispositif quelconque n'est ainsi plus réduit à un texte que l'usager doit lire pour l'utiliser, il permet [afford] au contraire un certain nombre d'utilisations. Ses affordances sont perçues par 
Retour sur une (d)ébauche :

Une problématique communicationnelle du changement technique

l'usager en tant que "propriétés physiques de sa substance et de ses surfaces" sans pour autant être des qualités intrinsèques du dispositif mais plutôt des propriétés émergentes de la perception du dispositif par l'usager. En ce sens l'affordance traduit une relation de l'usager au dispositif (Gibson, 1977).

Dans le cadre d'une conception rénovée qui substitue l'affordance à l'inscription, la notion de virtualité, entendue comme le continuum qui existe entre l'inscription et la présence concrète (les deux formes opposées de la présence stable, physique et symbolique) recouvre tout son domaine d'action : au pôle symbolique de l'inscription, supposant un code stable, est adjoint un pôle physique supposant la stabilité de la corporalité. La virtualité commence entre les deux, au niveau des interprétations symboliques du message non prévues par l'émetteur, jusqu'au niveau des affordances non perçues. Plus encore, l'acteur virtuel, maintenant si souvent présent dans nos pratiques de communication, se caractérise par la somme des affordances qu'il a rendu possible dans le dispositif, que ces possibilités existent au niveau du design du dispositif en tant que modèle de l'usager ou en tant que limites à l'usage. Ceci m'a permis de proposer un modèle englobant centré sur le dialogue concepteurusager.

À l'origine du processus innovant, l'usager n'est que virtualité, ensemble de représentations abstraites dans l'univers mental du concepteur. Puis, à mesure de la construction du réseau socio-technique, l'usager prend plusieurs visages qui entrent en compétition pour une définition plus ou moins hégémonique de l'usage. L'usager est un construit mental qui s'actualise progressivement alors que les concepteurs (de même que la plupart des autres acteurs impliqués) tentent de parler en son nom, de le représenter après se l'être représenté. Chaque acteur impliqué dans le développement technologique - en défendant ses positions au sein de controverses concernant la définition de l'objet technique - participe ainsi à la délimitation du champ des usages et usagers possibles de la nouvelle technologie.

Comment les représentations des concepteurs sont-elles à l'origine des modes de configuration de l'usager ? La notion de configuration implique un certain arrangement d'éléments opérationnels ou fonctionnels. Une configuration renvoie donc à une représentation active, orientée vers un but : elle n'est pas rêve, mais bien fiction opérationnelle (Woolgar, 1991). II s'agit donc d'un terme de passage, d'un processus d'actualisation : les représentations des concepteurs s'opérationnalisent en configurations qui 


\section{Thierry BARDINI}

actualisent l'usager, en se différenciant au contact du réel. À ce titre, les configurations ont une valeur programmatique ; prises dans leur ensemble, elles définissent un programme d'action, celui des concepteurs vis-à-vis des usagers en devenir. Par ailleurs, ce processus d'actualisation est concomitant à un processus de réalisation de l'usager. En effet, l'imaginaire des concepteurs est nourri par leurs traductions de pratiques réelles co-existantes, qui signifient autant de possibles relais ou d'ancrages effectifs pour leur programmation. Dans ce sens, les prescriptions et proscriptions synthétisées dans les configurations de l'usager résultent aussi des descriptions des pratiques concrètes correspondantes, mises en oeuvre dans les phases de test ou bien encore par analogie. Au total, l'imaginaire des concepteurs, source de représentations, s'actualise en dispositifs socio-techniques configurant-c'est-à-dire contraignant et habilitant - l'usager. Comme le dit Gilles Deleuze, «le propre de la virtualité, c'est d'exister de telle façon qu'elle s'actualise en se différenciant, et qu'elle est forcée de se différencier, de créer ses lignes de différenciation pour s'actualiser » (Deleuze, 1966, p. 100).

\section{Quelques pistes problématiques}

Le terme «innovation », comme tous les substantifs en -tion dérivés de verbes d'action, désigne à la fois un produit et le processus qui génère ce produit. Ainsi, par exemple, un ordinateur est une innovation, mais le processus - ou la décision - qui préside à son introduction au sein d'une organisation est lui-même une innovation. Nous sommes donc toujours confrontés à deux faces d'une même réalité : d'une part la production et de l'autre la diffusion. Trois définitions classiques de l'innovation permettent de préciser cette dualité.

- La définition de Rogers (1995) insiste sur la perception de la nouveauté plutôt que sur une nouveauté absolue, en définissant l'innovation comme «une idée, une pratique, ou un objet perçu comme nouveau par un individu ou une autre unité d'adoption. » (p.11)

- La définition de l'Encyclopaedia Universalis insiste sur le caractère téléologique du processus d'innovation, en caractérisant l'innovation comme «tout changement introduit dans l'économie par un agent quelconque et qui se traduit par une utilisation plus efficiente des ressources. » (2004, p.530)

- La définition de Kanter (1988) insiste sur la dualité des niveaux d'organisation inscrite dans la dynamique d'innovation (le processus) : « une série de tâches menées à un niveau micro par des individus 
Retour sur une (d)ébauche :

Une problématique communicationnelle du changement technique

ou des groupes d'individus à l'intérieur d'une organisation. Ces processus sont à leur tour stimulés, facilités et renforcés- ou l'inverse- par une série de conditions au niveau macro. » (p.171)

$\mathrm{Au}$ regard de ces définitions provisoires et de mes travaux concernant l'analyse socio-historique du processus d'innovation dans le cas de l'informatique personnelle aux États-Unis (Bardini, 2000), je propose une problématisation du changement technique et social en huit propositions. Je vais maintenant les résumer rapidement.

\section{L'innovation comme tissu de médiations entre concepteurs et usagers}

Les opérations de médiations inscrites dans un dispositif technique quelconque traduisent un ensemble de relations liant deux types centraux d'acteurs, désignés par les vocables de « concepteurs » et « d' usagers ». II n'y a pas une innovation qui apparaît ex-nihilo et dont, par la suite, les promoteurs déploient un réseau de communication pour se chercher des usagers : l'usager fait partie intégrante du dispositif, il est une construction socio-technique au même titre que le produit dont il apparaît comme une sorte d'ombre portée. L'implication des usagers débute dès l'origine du processus d'innovation, sous la forme de fictions opérationnelles où ils apparaissent en tant que représentations. Mes travaux sur la genèse de l'informatique personnelle ont montré ce premier point en insistant sur le fait que les premières représentations de l'usager sont réflexives, image inverse du concepteur dans le miroir de son projet.

\section{L'innovation implique des acteurs plus ou moins virtuels}

Le développement d'un dispositif socio-technique met en scène (présente et re-présente) des acteurs plus ou moins virtuels, au sens que leur présence effective décrit un continuum qui va de la présence physique et concrète jusqu'à la présence sous forme d'inscriptions stables et consensuelles dans le dispositif ; Tous les acteurs ne sont évidemment pas présents au même titre durant chaque phase du processus d'innovation. Ainsi, au moment de la conception de l'innovation, l'usager apparaît souvent comme une virtualité, une représentation plus ou moins imaginée par les concepteurs ou extrapolée à partir de leur analyse de la population qu'ils ciblent pour leur dispositif ou produit. L'implication des usagers commence dès ce moment et il faut porter une attention particulière 
Thierry BARDINI

aux justifications de cette implication par les concepteurs, qui s'érigent alors en représentants des usagers à venir. Ces justifications sont inscrites dans le dispositif en fonction de négociations entre concepteurs.

\section{Les modalités de présence des acteurs sont incertaines}

Le cycle de vie d'un dispositif socio-technique présente une source d'incertitude majeure, celle qui concerne les modes de présence des acteurs et des conditions de passage d'un mode de présence à un autre. Les acteurs changent de modalité de présence durant le cycle de vie de l'innovation, qui va de sa conception à l'usage massif du dispositif : I'usager s'actualise peu à peu, tandis que le concepteur se virtualise. De représentation dans l'esprit du concepteur, l'usager devient réel au fur et à mesure qu'émergent dans les négociations des représentants légitimés par leur pratique de l'innovation. À l'inverse, les concepteurs se virtualisent et disparaissent des négociations pour n'apparaître finalement qu'à l'état de traces (ou affordances pour l'usager) dans le dispositif témoignant des décisions de conception qu'ils ont prises.

\section{La représentation suppose la délégation}

On appelle délégation la situation ou l'un quelconque des acteurs accepte d'être représenté dans le dispositif socio-technique par toute autre entité qui agit à sa place. La délégation n'est jamais absolument définitive et la virtualité de l'acteur qui délègue suppose qu'il lui est possible à tous moments de ré-ouvrir les négociations sur la représentativité de l'entité à laquelle il délègue. La controverse suppose donc un changement des conditions de la présence de l'acteur qui la crée ; Le mécanisme élémentaire du processus est la délégation, lorsque l'un quelconque des acteurs disparaît virtuellement du dispositif pour y être remplacé par une autre entité qui le représente (cette autre entité n'étant pas nécessairement humaine). Le cycle de vie du dispositif ou du produit est marqué par des controverses à chaque fois que la représentativité de l'une ou plusieurs de ces entités est remise en question. Lors de la controverse, l'acteur qui a délégué re-apparaît dans les négociations.

\section{La stabilité du dispositif ou du produit dépend du consensus sur les opérations de délégation}

En l'absence de controverse, le dispositif est quasi-stable, ce qui signifie en pratique que les associations permises par les opérations de délégation reposent sur un consensus suffisamment stable pour que l'on puisse le considérer comme « inscrit» dans le dispositif. Le cycle de vie du dispositif 
Retour sur une (d)ébauche :

Une problématique communicationnelle du changement technique

présente donc des phases de stabilité où les opérations de délégation fonctionnent et la présence précédente des acteurs ayant délégué est traduite en inscriptions dans le dispositif. Le caractère consensuel de cette stabilité est fondamental, et le recours à la coercition pour l'établir ne peut être que restreint, même si il est parfois nécessaire. Le contrôle des inscriptions doit en principe reposer sur la coopération des acteurs qui acceptent leur virtualité.

\section{La stabilité du dispositif est une condition nécessaire à sa diffusion}

La diffusion du dispositif ou du produit dans une population croissante d'usagers suppose la stabilisation plus ou moins complète, mais jamais absolument réalisable, de celui-ci. L'apparition de la masse critique dans la trajectoire de diffusion dépend donc en dernière analyse de l'inscription consensuelle d'opérations de délégation dans le dispositif socio-technique luimême. La masse critique est définie comme le nombre minimum d'adopteurs requis pour maintenir le processus de diffusion (Valente, 1995). C'est une mesure au niveau de la population-cible, utilisée par analogie avec les notions de masse critique en physique nucléaire et en épidémiologie, qui mettent l'accent sur le fait qu'une fois atteinte la masse critique, les phénomènes sont incontrôlables et inexorables. Atteindre la masse critique est souvent présenté comme l'objectif à atteindre pour les agents en charge de gérer ou de promouvoir une innovation.

\section{La masse critique dépend de la structure des paliers d'adoption de la population cible}

Le pallier d'adoption d'un usager donné caractérise sa réaction individuelle vis-à-vis du consensus inscrit dans le dispositif. L'apparition de la masse critique est directement déterminée par la structure des paliers d'adoption de la population d'usagers. Le seuil d'adoption d'un individu est défini comme la proportion d'adopteurs dans son réseau personnel nécessaire pour le ou la convaincre d'adopter à son tour (Valente, 1995). Vis-à-vis des caractérisations antérieures des adopteurs envisagés sur la base uniquement « psychologique » de leur réaction à l'influence (inovativness ou «propension à innover»), l'introduction du seuil d'adoption a le mérite de faire intervenir dans l'analyse des variables positionnelles, qui rendent compte de l'intégration des adopteurs dans les réseaux de la diffusion de l'innovation. Une hypothèse de normalité de 


\section{Thierry BARDINI}

la distribution des seuils d'influence reproduit les postulats du modèle standard de la diffusion des innovations tandis que l'analyse des réseaux personnels des adopteurs permet une analyse sociologique plus approfondie.

\section{L'interface des réseaux du développement et de la diffusion}

Pour interpréter le relatif succès de la diffusion de masse d'une innovation, il convient donc d'expliciter la phase qui va du moment de l'interface entre les réseaux de sa conception et ceux de sa diffusion jusqu'à l'apparition de la masse critique. Le critère analytique central pour cette description réside dans la relative stabilité du dispositif socio-technique au regard des chaînes d'associations entre toutes les entités qui le composent. La masse critique, synonyme de succès de l'innovation, apparaîtra d'autant plus vite que les acteurs-usagers à faibles seuils d'adoption sont centraux dans les réseaux de la diffusion et que les associations entre acteurs du développement de l'innovation résistent à toute tentative de traduction concurrente qui re-ouvrirait les négociations, ou en d'autres termes, que la stabilité du dispositif rend difficile l'apparition de toute controverse subséquente. II existe donc bien un lien entre les réseaux du développement et ceux de la diffusion de l'innovation : celui que constituent ces individus représentatifs d'usagers potentiels pour la phase de développement et plus ou moins influents dans les réseaux personnels de la diffusion. En clair, les représentants des usagers des dernières phases du développement (et en particulier des phases de test) doivent se muer en pairs influents lors de la phase de diffusion. Leur médiation, comme toutes celles qui constituent le script du dispositif, perdure à l'innovation, elle perdure même à l'adoption de l'innovation. Toute forme d'organisation efficace à un moment donné du cycle de vie ne peut en aucun cas être tenue pour absolument efficace et permanente.

Au total, il apparaît donc que les réseaux de la propagation des influences traduisant la diffusion d'une innovation dans une population-cible sont des structures instables et dynamiques, et que leur activité ne s'arrête pas au moment où le comportement d'adoption est observé, même pour un nombre d'individus correspondant à la masse critique : la médiation perdure à l'innovation, elle perdure même à l'adoption de l'innovation. Les problématiques de l'appropriation rendent comptent de ces médiations qui durent. Le coeur de l'effort de gestion du processus d'innovation concerne l'incertitude le caractérisant, et ce à toutes les phases du cycle de vie du dispositif ou produit. Toute forme d'organisation efficace à un moment donné du cycle de vie ne peut en aucun cas être tenue pour absolument efficace et permanente. Pour 
Retour sur une (d)ébauche :

Une problématique communicationnelle du changement technique

résumer, en parodiant Jean Cocteau, la conjugaison du verbe innover ressemble à celle du verbe aimer : son passé n'est jamais simple, son présent n'est qu'indicatif, et son futur conditionnel.

J'insiste ici sur le fait que les propositions précédentes forment une problématique d'ensemble sur le changement technique et social plutôt qu'un modèle. Certaines des apories décelées antérieurement dans la critique des deux théories fondatrices persistent, inchangées. C'est en particulier le cas de ce que j'ai qualifié antérieurement de «quatrième brêche » dans l'édifice théorique de l'acteur-réseau: la problématique de la référence, c'est-à-dire la question des rapports entre les états du monde et leurs descriptions sémiotiques. À ce sujet, deux phases de crise distinctes m'ont finalement mené à remettre encore plus profondément en question l'ancrage de mes travaux dans la théorisation de l'acteur-réseau.

\section{Retour sur l'affaire Sokal et résurrection de la critique}

La première crise a été appelée « l'affaire Sokal ». Au printemps 1996, Alan Sokal, un physicien de l'Université de New York, publiait un papier intitulé "Transgressing the Boundaries: Toward a Transformative Hermeneutics of Quantum Gravity" dans la revue Social Text. Au moment de la parution de ce texte, Sokal annonçait dans un autre papier intitulé "A Physicist Experiments with Cultural Studies" (1996b) que l'article qu'il publiait dans Social Text était en fait une parodie, destinée à dénoncer «la prolifération de la pensée vague et absurde qui nie l'existence de réalités objectives » (ma traduction). Sokal capitalisait ensuite (1997) sur cette expérience en publiant, en collaboration avec Jean Bricmont, un livre intitulé Impostures Intellectuelles.

Avec sa parodie de texte de sociologie de la science, Alan Sokal a (re)posé (à son insu) la question cruciale qui hante de nombreux sociologues ayant participé à l'expérience constructiviste : quelles sont les conditions de plausibilité d'un texte sociologique ? Évidemment, les sociologues en question n'étant pas exactement des idiots philosophiques, cette question était déjà posée, elle l'était même depuis le texte fondateur du mouvement, qualifié de programme fort en sociologie de la science. Mais la stratégie de Sokal pour la poser à nouveau était intéressante à deux titres au moins : (1) d'abord parce que Sokal est un 


\section{Thierry BARDINI}

physicien, c'est-à-dire un scientifique, et ensuite (2) parce que Sokal a posé la question pratiquement, en écrivant un « texte de sociologie de la science ».

Lorsque les sociologues constructivistes disaient que les scientifiques sont souvent de bons sociologues, ils voulaient dire en fait que puisque la science est aussi une construction sociale (une expérience collective), les scientifiques doivent démontrer une certaine intelligence du social pour espérer réussir dans leurs entreprises. Voila maintenant qu'un scientifique allait un peu plus loin, en démontrant apparemment un talent certain dans la pratique professionnelle de la sociologie. Non seulement, il démontrait une certaine intelligence du social, mais il était en plus capable d'écrire un texte validé par les professionnels de la profession sociologique. Quel scandale ! Or qu'est ce qu'a démontré Sokal en l'occurrence, si ce n'est sa capacité à intégrer les codes de la production littéraire dite sociologique?

Ceci n'a rien de révolutionnaire en soi lorsqu'on prend soin de rappeler que cette sociologie constructiviste est d'abord le fait d'une génération nouvelle de sociologues émergeant au début des années soixante-dix et incluant dans ses rangs de nombreux scientifiques. II est peut-être nécessaire de rappeler que la brèche fut ouverte par le texte fondamental de Thomas Kuhn, La structure des révolutions scientifiques, et que Kuhn était un physicien. Dans cette perspective, le fait le plus intéressant n'est pas que Sokal ait réussi à écrire un texte sociologique-il a seulement montré que cela ne suppose aucune intelligence autre que celle des codes littéraires de la sociologie professionnelle. Mais bien plutôt que ce faisant, il ait redéfini les critères minimaux de ce qui peut être considéré comme un texte sociologique : une apparence de sociologie encodée en mots.

Car enfin qu'est ce qu'a fait Sokal si ce n'est singer le sociologue dans sa production littéraire et ainsi poser la question cruciale : la sociologie peut-elle donc se réduire à une production littéraire d'un certain type ? Le travail du sociologue peut-il donc se passer de ce qui précède l'écriture ? Le sociologue n'est-il donc que le créateur d'un certain type de discours, conventionnellement calibré? Le tournant sémiotique peut-il faire fi de toute problématique de la référence ? Si Sokal a en effet enfoncé le dernier clou dans le cercueil de la sociologie constructiviste, c'est bien que parce que lesdits sociologues ont été incapables de lui répondre sur ce terrain quoi que se soit de signifiant. Et pour cause ! Sokal les a en effet placés face à une double contrainte fatale : lui donner raison et accepter que la sociologie constructiviste se résume à une production de discours conventionnellement tenus pour sociologiques (à moins que ce ne soit pour constructivistes), ou tenter de l'infirmer, ce qui reviendrait en 
Retour sur une (d)ébauche :

Une problématique communicationnelle du changement technique

fait à aller jusqu'au bout d'une explicitation méthodologique qui infirmerait en retour les bases mêmes de la démarche sociologique constructiviste. Latour (1988) n'avait-il pas jadis proposé de «remplacer la méthodologie par le style »?

Plus encore, cette affaire a achevé de me convaincre que les technologies littéraires, si cruciales pour l'avènement de la science expérimentale, ne sont plus qu'une partie des technologies disponibles pour décrire le monde. Or ce monde décrit a profondément changé avec l'apparition de nouveaux modes de rapport, certes produits par la technoscience, mais maintenant relativement ouverts à d'autres initiatives que celles des scientifiques et ingénieurs. L'univers machinique de la simulation est ce contexte supérieur. La machine spectaculaire, c'est-à-dire la machine à produire des représentations, est littéralement en train de faire exploser le mythe de la personne humaine. J'ai bien peur que suite à son tournant sémiotique, la sociologie constructiviste n'ait rien à dire à ce sujet, si ce n'est offrir ses propres représentations désincarnées, et prêter ainsi encore plus le flanc à l'argument du tu quoque. Quelque part à la fin du millénaire, la question de la critique, évacuée de mon travail depuis mon éducation marxisante mal digérée, est revenue en force. Une sorte de vengeance du refoulé qui m'a finalement conduit à rompre définitivement avec les positions (et les postures) de l'acteur-réseau.

La deuxième crise, terminale celle-ci, est advenue suite à la lecture d'un papier de Bruno Latour. II est des textes qui, tout en me mettant dans une colère noire, peuvent m'inspirer profondément. La conférence de Bruno Latour, intitulée « Why has Critique Run out of Steam. From Matters of Fact to Matters of Concern » donnée à Stanford en avril 2003, discutée à Harvard durant le premier semestre de 2004 pour être finalement publiée cette même année dans Critical Inquiry est de ce type.

Dans ce texte, Latour caricature la pensée critique sous une forme maintenant classique : ils disent qu'ils savent plus que les gens car ils pensent que ceux-ci vivent dans l'illusion complète des motifs réels (de leurs actions et idées), ils en appellent à des agences invisibles, ils sont des théoriciens de la conspiration. Du fait de l'actualité, si pressante, il faut poser un constat: une certaine forme d'esprit critique a conduit à cette impasse. Plus encore, c'est elle, cette certaine forme d'esprit, qui nous a conduit à combattre l'empirisme alors qu'il fallait le renouveler. Mais rassurez vous, l'empirisme n'est pas mort, et Latour se propose de le ressusciter pour nous. Pour cela, il est prêt à renoncer 


\section{Thierry BARDINI}

aux matters of fact, chers aux Science Studies, pour se pencher sur de plus pressantes matters of concern, en se posant la question nécessaire : comment rendre leur objectivité aux choses?

Mais d'où viennent donc ces matters of concern ? La réponse n'est pas évidente à la lecture du texte de Latour, mais Isabelle Stengers, qui en est l'inspiration principale, la donne dans son livre sur Whitehead, lorsqu'elle cite (2002, p. 493) un extrait de Modes of Thought où Whitethead précise que ce terme doit être entendu dans son sens quaker. Vérification faite, Whitehead précise ceci à chaque fois qu'il utilise ce terme. Les quakers considèrent le concern comme une croisade personnelle de l'individu qui la présente devant son groupe (ou gathering) pour qu'elle y soit testée. Si elle n'est pas retenue par le groupe, le concern est qualifié de notion, entendue péjorativement comme toute direction religieuse ou spirituelle non pertinente. Ainsi entendu, le concern est bien une construction collective issue de la lumière intérieure d'un individu singulier dans son rapport à Dieu. Stengers a donc raison lorsqu'elle considère Whitehead comme un agnostique constructiviste : « son agnosticisme est constructiviste car la manière dont la religion est décrite engage celui qui la décrit, implique un pari sur ce qui peut 'faire tenir' une religion, sur ce dont pourrait être capable le sentiment religieux »(p. 156). Voila ce qui semble convenir aux positions préalables de Latour, mais nie quelque peu la portée du virage qu'il propose de prendre, des matters of fact vers les matters of concern. En effet, si l'on croit cette référence, il semblerait bien que ces derniers puissent être traités, épistémologiquement et méthodologiquement, comme les bons vieux matters of fact des Science Studies : de manière agnostique et constructiviste, mais personnelle...

Bruno Latour, encore une fois, brûle un épouvantail qu'il a construit luimême. La diffusion des innovations a d'abord joué ce rôle-dans une version obsolète de l'avis même des diffusionnistes. En cela, mon texte de 1993 le suivait encore, tout en tentant de dire que la théorie de la diffusion des innovations avait été amendée de l'intérieur dans un sens plus proche de ce que les constructivistes tenaient pour essentiel. Maintenant, tel son Janus inaugural (Latour, 1987), l'épouvantail est doté de deux têtes : la vieille critique, de Marx à Baudrillard en passant par Bourdieu (les théoriciens de la conspiration), mais aussi la sociologie de la science constructiviste elle-même, maintenant rendue obsolète elle aussi. Dans ce texte, Latour écrit: «cela ne veut pas dire que nous ayons eu tort mais simplement que l'histoire change rapidement et qu'il n'y a pas de pire crime intellectuel que de considérer avec l'équipement d'une période révolue les défis du présent. » (2004, p.231). Malheureusement, la nouvelle manière d'avoir raison ressemble à s'y méprendre à celle supposément 
Retour sur une (d)ébauche :

Une problématique communicationnelle du changement technique

« obsolète », à ceci près qu'elle aura simplement permis d'annexer la critique au passage.

Par contre, aucune des apories et des impasses si clairement décrites durant les années 1990 n'ont été dépassées : matters of fact ou concerns s'inscrivent toujours dans un nominalisme radical et un empirisme qui les prolonge logiquement, mais qui n'expose finalement que leurs faiblesses méthodologiques. Ceci conduit les théoriciens de l'acteur-réseau à pratiquer l'acte de langage performatif plus souvent qu'à leurs tours. En 1995, alors que j'évoquai, dans un panel dirigé par Michel Callon d'une conférence à Hong Kong, les quelques critiques de l'acteur-réseau reprises dans le présent papier, il resta singulièrement silencieux. Ce n'est que dehors qu'il me répondit que j'avais certainement raison mais que mes critiques dataient et que depuis quelques années ils avaient trouvé la solution pour sortir de ces impasses. Quelle solution ? Callon se garda bien de me l'indiquer. L'argument d'autorité constitue peut-être, après tout, cette solution. Le matter of concern, concept vague destiné à ressusciter la critique, n'est-il finalement autre chose que la justification arbitraire d'un professionnel du debunking déclarant que cela nous concerne puisqu'il le dit?

Pour ma part, j'ai alors commencé à chercher ailleurs. Où et quoi, serait une autre histoire...

À Everret M. Rogers, In memoriam 
Thierry BARDINI

\section{Références bibliographiques}

AKRICH M., 1992, «The De-Scription of Technical Objects », dans W. E. BIJKER et J. LAW (dir), Shaping Technology/ Building Society, pp.205-224, Cambridge (MA), MIT Press.

AKRICH M., 1993, « Les objets techniques et leurs utilisateurs. De la conception à l'action, » Raisons Pratiques, $n^{\circ} 4$, pp.35-57.

ASHMORE M., 1989, The Reflexive Thesis. Wrighting Sociology Scientific Knowledge, Chicago, University of Chicago Press

BARDINI T., 1993, «Diffusionnisme, Constructivisme et Modèle Technique : Ébauche d'une Approche Communicationnelle du Changement Technique », Technologies de l'Information et Société, vol.5, n4, pp.367-391.

BARDINI T., 1996, « Réseaux et changement socio-technique : de l'inscription à l'affordance », Réseaux, n76, pp.63-93.

BARDINI T., 2000, Bootstrapping: Douglas Engelbart, Coevolution, and the Origins of Personal Computing, Stanford (CA), Stanford University Press.

BLOOR D., 1976, Knowledge and social imagery, Londres, Routledge.

CALLON M. et B. LATOUR, 1992, « Don't Throw the Baby Out with the Bath School! A Reply to Collins and Yearly » dans A. PICKERING (dir), Science as Practice and Culture Chicago, The University of Chicago Press, pp.343368.

CHAMBAT P., 1994a, « Usages des technologies de l'information et de la communication: évolution des problématiques », Technologies de l'information et société, vol.6, n³, pp.249-269.

CHAMBAT P., 1994b, «NTIC et représentations des usagers » dans A. VITALIS (dir), Médias et nouvelles technologies, Rennes, Apogée, pp.45-59.

COLLINS H. M. et S. YEARLEY, 1992a, « Epistemological Chicken » dans A. PICKERING (dir), Science as Practice and Culture, Chicago, The University of Chicago Press, pp.301-326.

COLLINS H. M. et S. YEARLEY,1992b, « Journey into Space », dans A. PICKERING (dir), Science as Practice and Culture, Chicago, The University of Chicago Press, pp.369-389.

DELEUZE G., 1966, Le bergsonisme, Paris, Presses Universitaires de France. 
Retour sur une (d)ébauche :

Une problématique communicationnelle du changement technique

GIBSON J. J., 1977, "The Theory of Affordances », dans R.SHAW et J. BRANSFORD (dir), Perceiving, Acting, and Knowing. Toward an Ecological Psychology ${ }_{2}$ Hillsdale (NJ), Lawrence Erlbaum, pp.67-82.

JOUËT J., 2000, «Retour critique sur la sociologie des usages », Réseaux, $\mathrm{n}^{\circ} 100, \mathrm{pp} .487-521$.

KANTER R. M., 1988, « When a thousand flowers bloom: Structural, collective and social conditions for innovation in organisation », Research in organisational behavior, $\mathrm{n}^{\circ} 10, \mathrm{pp} .169-211$.

KUHN T. S., 1970, La structure des révolutions scientifiques, Chicago, University of Chicago Press, seconde édition, Paris, Flammarion, 1983, pour la traduction française.

LATOUR B., 1983, «Comment redistribuer le grand partage ? » Revue de synthèse, CIV, n¹10, pp.203-236.

LATOUR B., 1987, Science in Action, Cambridge (MA), Harvard University Press.

LATOUR B., 1988, "The Politics of Explanation: an Alternative », dans S. WOOLGAR (dir), Knowledge and Reflexivity. New Frontiers in the Sociology of Knowledge, Newbury Park (CA), Sage, pp.155-177.

LATOUR B., 2004, « Why has Critique Run out of steam? From Matter of Facts to Matters of Concern », Critical Inquiry, vol.30, n², pp.225-236.

LAW J., 1987, «Technology and Heterogenous Engineering: The Case of Portuguese Expansion. » dans W. BIJKER, T. P. HUGHES et T. PINCH (dir), The Social Construction of Technological Systems, Cambridge: MIT Press, pp.111-134.

LENOIR T., 1994, « Was the Last Turn the Right Turn? The Semiotic Turn and A. J. Greimas », Configurations ${ }_{1}$ vol.2, n¹, pp.119-136.

MILLERAND F., 1999, «Usages des NTIC : les approches de la diffusion, de l'innovation et de l'appropriation, 2e partie : l'appropriation », Commposite, v.99.1, <http://commposite.org/v1/99.1/articles/ntic_2.htm >, dernière consultation le 14 janvier 2008.

PICKERING A. (dir), 1992, Science as Practice and Culture, Chicago, University of Chicago Press. 
Thierry BARDINI

RICE R. E. et E. M. ROGERS, 1980, « Re-invention in the innovation process », Knowledge : creation, diffusion, utilization, vol.1, $\mathrm{n}^{\circ} 4$, pp.499-514.

ROGERS E. M., 1995, Diffusion of innovations, quatrième édition, New York, Free Press.

SOKAL A., 1996a, «Transgressing the Boundaries: Toward a Transformative Hermeneutics of Quantum Gravity » Social Text, n46/47, pp.217-252.

SOKAL A., 1996b, «A Physicist Experiments with Cultural Studies », Lingua Franca, vol.6, $\mathrm{n}^{\circ} 4$, pp 62-64.

SOKAL A. et J. BRICMONT, 1997, Impostures intellectuelles, Paris, Odile Jacob,.

STENGERS I., 2002, Penser avec Whitehead, Paris, Le Seuil.

TAYLOR J. R., F. COOREN, N. GIROUX et D. ROBICHAUD, 1996, «The communicational basis of organization: Between the conversation and the text », dans Communication Theory, vol.6, n¹, pp.1-39.

VALENTE T., W, 1995, Thresholds Models of the Diffusion of Innovations, Cresskill (NJ), Hampton Press.

VEDEL T., 1994, «Sociologie des innovations technologiques et usagers: introduction à une socio-politique des usages » dans A. Vitalis (dir), Médias et nouvelles technologies, Rennes, Apogée, pp.13-34.

VON HIPPEL E., 1986, « Lead Users: A Source of Novel Product Concepts. » Management Science, vol.32, $\mathrm{n}^{\circ} 7, \mathrm{pp} .791-805$.

WHITEHEAD A. N., 1933 (1967), Adventures of Ideas, New York, Free Press.

WHITEHEAD A. N., 1938 (1968), Modes of Thought. New York, Free Press.

WOOLGAR S., 1991, «Configuring The User: The Case of Usability Trials », dans J. LAW (dir), A Sociology of Monsters, Londres, Routledge, pp.57-99.

\section{Pour citer cet article :}

BARDINI, T., «Retour sur une (d)ébauche : une problématique communicationnelle du changement technique», Revue tic\&société [En ligne], Volume 1, $n^{\circ} 1,2$ ème semestre 2007, mis à jour le : 15/02/2008, URL :

<http://revues.mshparisnord.org/ticsociete/index.php?id=245>. 